\title{
El comportamiento escolar basado en la relación parental en estudiantes de idioma extranjero
}

Danny Hallo

Universidad Regional Autónoma de los Andes

ur.dannyhallo@uniandes.edu.ec https://orcid.org/0000-0002-0315-8669

Giomara Quizhpe Universidad de Guayaquil giomy.quizhpe@gmail.com https://orcid.org/0000-0002-6261-947X

Carlos Taco

Universidad Estatal de Bolívar carlostacot@yahoo.com.mx https://orcid.org/0000-0002-4471-4975

\section{Resumen}

Los estudiantes son influidos por varios factores externos, como sociales, biológicos, familiares e intelectuales, algunos de los factores pueden incidir de forma directa o incluso indirectamente en el comportamiento de los estudiantes, en el aprendizaje, en el accionar etc., los seres humanos por naturaleza son entes de constante relación en los ambientes en donde existe interacción, por ello, la presente investigación tiene como fin, comprender los problemas de comportamiento y los estilos de 
socialización parental en el aula de estudiantes de la modalidad abierta del idioma ingles nivel B1+, la metodología de investigación aplicada es de enfoque cualitativo, con tipos de investigación bibliográfico documental y de campo, además se contó con un alcance descriptivo, como instrumentos de evaluación, se utilizó ESPA 29 para conocer los estilos parentales y el test TOCA-R para identificar la presencia de problemas de comportamiento, los resultados obtenidos en el estudio fue la prevalencia del estilo autorizativo en madres y padres, a pesar de ello, no existe problemas de comportamiento en la mayoría de evaluados, se concluye que existe presencia mínima de problemas aceptación de autoridad y de contacto social.

Palabras clave: Relación parental, estilos parentales, familia, comportamiento, estudiantes.

\begin{abstract}
Students are influenced by various external factors, such as social, biological, family and intellectual, some of the factors can directly or even indirectly influence student behavior, learning, action etc., human beings by nature they are entities of constant relationship in the environments where there is interaction, therefore, this research aims to understand behavior problems and parental socialization styles in the classroom of students of the open modality of the English language level B1 + , the applied research methodology is of a qualitative approach, with types of documentary and field bibliographic research, in addition there was a descriptive scope, as evaluation instruments, ESPA 29 was used to know the parental styles and the TOCA-R test for identify the presence of behavioral problems, the results obtained in the study were and the prevalence of the authorizing style in mothers and fathers, despite this, there are no behavioral problems in the majority of those evaluated, it is concluded that there is minimal presence of problems of acceptance of authority and social contact.
\end{abstract}

Keywords: Parental relationship, parental styles, family, behavior, students. 


\section{INTRODUCCIÓN}

La funcionalidad familiar va más allá de resguardar la supervivencia y el estado físico, ya que involucra la promoción del desarrollo familiar en todos los aspectos como en el ámbito afectivo y social de los hijos, que trascenderá hasta la edad adulta; por esta razón, las relaciones familiares que se presenten tendrán incidirán en el desarrollo. Así como también, varios autores opinan que existe una relación bidireccional entre hijos y padres, considerando, que los hijos ya no mantienen únicamente con el rol como espectadores pasivos, sino que también intervienen en la interacción familiar y en las relaciones que mantienen con sus padres (Ortega, Plancarte, Garrido, Reyes, \& Torres, 2018). La interacción familiar nace como un conjunto de relaciones en donde interviene la colaboración, reciprocidad, poder e incluso los conflictos que surgen en el interior de las familias, sin embargo, la pareja representa la parte central de la estructura familiar. (Chávez, 2013).

Los seres humanos son personas sociales, por ello, mantienen una interacción constante, los individuos centran su primera interacción con el ambiente familiar, es así los miembros de la familia se encuentra en un proceso continuo de socialización, considerando de más relevancia la relación de padres e hijos. Los padres mantiene un rol relevante en la vida de sus hijos, son considerados como ejemplo y motivación, en los aspectos que abarca el contexto social, educativo, entre otros., pasan por la opinión familiar, como consecuencia estas acciones pueden influenciar y generar aspectos efectos positivos y negativos (Oliva \& Villa, 2014).

En lo descrito por Aguinaga, Rimari, \& Valázquez (2018) las funciones que deben cumplir los padres son incalculables, entre una de las principales se encuentra la socialización, función que involucra varios aspectos, como es la guía de una buena sociabilización, es importante considerar que la responsabilidad de esta función es colectiva entre la misma sociedad y el ambiente en donde se desarrolla la familia.

En lo descrito por C. Espinoza \& Panta(2014) un estilo parental se define como una estrategia que los progenitores aplican en la crianza, cuidado, enseñanza, protección y todos los aspectos referente a los hijos, los estilos tienen la finalidad de cumplir objetivos específicos relacionados a los deberes y funciones de los padres

El paso de la etapa de la adolescencia a la etapa de juventud es una etapa en la cual los jóvenes obtienen responsabilidades y obligaciones diferentes generando cambios en su vida. Entonces, los jóvenes en el ámbito educativo desarrollan relaciones interpersonales, consiste en instaurar su propia identidad a través de la pertinencia e integración grupal. El rol que cumplen los padres es un factor principal para la guía de una joven, aspecto que se desarrolla en relación a la crianza, educación, confianza, u otros aspectos que fomenta la familia. (Brea, 2014)

En lo descrito por Espinoza (2015) La funcionalidad familiar en Ecuador se encuentra debilitado, los padres de familia tiene la función de fortalecer a la familia. Los jóvenes, en la mayoría de los casos tienen una percepción de sus padres en diferentes estilos; se comprende que los hijos reflejan lo que son sus padres al tomar como ejemplo lo realizado por lo mismo (Pérez, 2013)

El comportamiento de los estudiantes no adecuado en el aula es un problema común en el ámbito 
educativo, el $40 \%$ de problemas que se presentan en el aula son por dificultades de conducta, atención, baja adaptabilidad e impulsividad, los problemas conductuales se manifiesta por la complejidad y expresión de patrones de comportamiento negativos, desafiante y disocial, (Erazo, 2016). Por ello, padres, autoridades, maestros y compañeros buscan vías de solución para la problemática conductuales que se presentan.

Considerando a los problemas de comportamiento desde un aspecto global, la educación implica el efecto en el grupo escolar y no solo desde el individuo, el cambio se identifica en las interacciones entre compañeros, docentes y sobre todo familiares, se comprende como un conjunto de acciones de una persona y su efecto en otras personas, entendiendo así, que es un problema grupal y no individual (Gotzens, Badía, Castelló, y Genovar, 2007).

El objetivo del estudio es comprender los estilos parentales que predominan en los estudiantes, de igual forma conocer, sobre los problemas de comportamiento, resultados que se plantearan en esta investigación.

\section{Relación parental}

La socialización parental, se define como el proceso mediante el cual las personas adquieren normas, valores, creencias y diversas formas de conducta que son apropiadas en una sociedad (Torres, 2016)cross-cutting and correlation type, in order to determine if there is association between the varying styles of parental socialization and social skills in a sample consisting by 177 students of both genders from 12 to 18 years old in a Particular educational institution in Metropolitan Lima. Parental socialization styles questionnaire was used in adolescents (ESPA 29. En las etapas de infancia y adolescencia es fundamental el proceso de socialización, teniendo en cuenta que la familia es uno de los principios primordiales para la enseñanza de guías culturales. Tradicionalmente se entiende como dirección principal de la socialización la que se realiza de padres a hijos (Suárez \& Vélez, 2018).

Conforme ha pasado el tiempo la socialización dio un giro como proceso bidireccional, en el cual los padres están inmersos en un proceso de socialización por parte de los hijos, acorde al desarrollo la influencia está sujeta a varios agentes como los medios de comunicación, institución educativa y experiencias (Ortiz y Moreno, 2016). En base a lo expuesto hasta el momento el medio donde se desenvuelve el niño o adolescente, puede ser en un espacio o con individuos llega a ser vital, puesto que, llegar a ejercer influencia en el progreso de la relación parental.

En el año de 1966 surge la necesidad de comprender la relación parental, por esta razón, comienzan a presentarse investigaciones direccionadas a los estilos parentales, entendiendo los aspectos que comparten los padres hacia sus hijos a través de estrategias de crianza. Lo señalado se centra en la socialización, a causa de que es el medio de enseñanza para los padres y el comportamiento respaldándose por medio de las normas culturales y sociales.

De acuerdo a los expuesto por Córdoba (2014) la socialización es una acción de establecer límites, la familia tiene la obligación de socializar a sus hijos, de acuerdo a la cultura que 
promuevan los padres; en otras palabras, es el proceso mediante el cual se transfieren modelos de cultura a nuevas generaciones, y es fácil de reconocerlo en las creencias y cultura. Se tiene como objetivo en el proceso de socialización parental el controlar y desarrollar la conciencia los impulsos, ejerciendo la función del rol social, los autores puntualizan que el aprendizaje no es consciente para el adolescente o niño, ya que va asimilando los aspectos de cultura por medio de los cuales el individuo podrá adaptarse y desenvolverse en la sociedad, por lo tanto, la familia tiene como objetivo de ser el ente socializador

Los estilos parentales se consideran como actividades prácticas que tienen la capacidad de reducir las diversas prácticas parentales educativas, que en conjunto de dimensiones se conectan entre sí formando varias composiciones, formando los diferentes tipos de educación familiar (Á. Capano \& Ubach, 2013).

Conforme a Á. Capano \& Ubach (2013), puntualiza que el comportamiento de los padres se basa en dos dimensiones identificadas, las mismas permiten determinar la influencia para la formación de los hijos, relacionar el control y la aceptación permite el surgimiento de los tipos parentales, en sus inicios se estipuló los siguientes estilos: a) estilo autoritario, se caracteriza por ser exigente y controlador; b) estilo democrático o autorizativo, sostiene un equilibrio entre calidez y exigencia; y c) estilo permisivo, corresponde a una formación de calidad y de permisibilidad. La exposición de la tipología detallada en el presente párrafo fue planteada por Baumrind en 1966.

Con el paso de los años en 1983 se propuso cuatro estilos parentales en conformidad de la respuesta y la demanda como variables, donde se expone los siguientes estilos: a) autoritario en el que se define una respuesta baja y una demanda alta; b) permisivo, cuenta con una respuesta alta y una demanda baja; c) negligente, respuesta baja y demanda baja; y d) autorizativo, se diferencia por tener respuesta alta y demanda alta (Marmo, 2014).

Los Ejes de la socialización parental son implicación/aceptación, esta dimensión se centra en el diálogo el mismo que describe la aprobación y el afecto de los padres por medio del diálogo con los hijos, en este eje de socialización aparece la autonomía la misma que se crea cuando tanto padres como hijos están satisfechos con su relación familiar. Los padres con un bajo nivel de implicación/aceptación son aquellos que no llegarán a un dialogo y mostrarán indiferencia (López, 2014).

Desde otra perspectiva, la coerción/imposición implica la indiferencia y el dialogo, con el propósito de eliminar en sus hijos los comportamientos negativos, es la dimensión que se conforma a la realidad de los padres cuando los hijos no se adaptan a las normas impuestas por la familia, se acude a esta dimensión para no realizar conductas negativas, por lo tanto, la privación y coerción dependerá de la decisión de los padres en ser verbal o física, sin embargo está atribuida de un contenido emocional fuerte (Comino y Raya, 2014).

La agrupación de las dos dimensiones tuvo como consecuencia el surgimiento de 4 estilos diferentes de socialización, donde se plantea un modelo bidireccional de las dimensiones independientes. 
Estilo autorizativo, se especifica por una alta coerción/imposición y una alta aceptación/implicación, esencialmente se emplea el diálogo obteniendo en sus hijos unos comunicadores, razón por el cual este recurso es el medio ideal para llegar acuerdos, así como también escuchar y expresar opiniones (Fuentes, García, Gracia, y Alarcón, 2015).

Estilo indulgente, se caracteriza por una baja coerción/imposición y una alta aceptación/ implicación, como un medio de comunicación, utilizando el razonamiento como herramienta disciplinaria. Impedir asignar reglas antes de haber mantenido un dialogo de la situación, prevalece el afecto (Fuentes et al., 2015).

En los establecido por A. Capano, González, y Massonnier (2016) el estilo autoritario, se especifica por una alta coerción/imposición y una baja aceptación/implicación, las muestras de afecto por medio de los padres no existe y mucho menos se utiliza el dialogo, además llegan a ser muy limitadas las herramientas de comunicación. La pretensión de los padres es controlar la conducta de sus hijos a través de un sistema rígido basado en normas

Estilo negligente, se caracteriza por una baja coerción/imposición y una baja aceptación/ implicación, es evidente la escasa implicación de afecto, al mismo tiempo, llega a ser mínima la responsabilidad hacia el control de sus hijos, conforme a esto, no se constituye normas ni límites. Se considera como el estilo inconveniente, debido a que no satisface las necesidades de los hijos, al no ofrecer apoyo, interacción o afecto hacia sus hijos (A. Capano et al., 2016).

\section{Comportamiento en el aula}

El comportamiento se describe como la forma de proceder de los individuos o colectivos ante estímulos, ambientes relacionados con su entorno. Desde una perspectiva psicológica, el comportamiento es un proceso que comprende todas las actividades que una persona llega a desempeñar en el medio. Cuando un comportamiento en lugar de ser dinámico se fija en la persona, pasa de ser un comportamiento a ser conducta (Huerta, 2018)

El comportamiento de cada persona, se lo expone por el argumento en el que se desenvuelve. La conducta de los individuos varía conforme a la cultura y la situación a la que están inmersos para el desempeño de las actividades cotidianas. No obstante, los contextos no son fijos, son dinámicos concerniente a sus actores sociales y sus interacciones con otros individuos o incluso con el ambiente (Guevara, 2013). Por lo tanto, el comportamiento depende del mismo individuo y el entorno que decidió crear.

Existe diversos tipos de comportamiento, según las circunstancias se clasifican en, comportamiento consciente se lo ejecuta después de razonar una situación en particular. En otras palabras, pensar antes de reaccionar ante algo o alguien. Comportamiento inconsciente corresponde a un comportamiento automático, pues la persona no reflexiona sobre su modo de reaccionar ante una situación específica. Comportamiento privado se caracteriza por ser de carácter íntimo, es decir se lo manifiesta en el hogar o a solas. En este apartado, el individuo no está mostrándose para otras personas, por lo tanto, suele ser más espontáneo y natural. (Carvajal, 


\section{5)}

No se debe encasillar a un comportamiento como bueno malo, más bien llega a depender del contexto en el que se están desenvolviendo dichas acciones. No obstante, generalmente el no obedecer una orden, infringir la ley, etc., son comportamientos impropios y ello ocasiona castigos o sanciones por parte de la autoridad (jueces, maestros, padres, etc.).

No se puede denominar a un comportamiento como bueno malo, pues todo dependerá del contexto en el que se están desarrollando dichas acciones. Sin embargo, generalmente el desacatar una orden, infringir la ley, etc., son comportamiento inadecuados y ello conlleva castigos o sanciones por parte de la autoridad (maestros, jueces, padres, etc.).

Existe numerosos comportamientos que un estudiante puede mostrar, Adame, García y Gómez (2017) indica que en toda aula de clases existe un alumno con problemas de todo tipo, por ejemplo: llega a ser violento con sus compañeros o maestro, lanza los útiles escolares, no tiene ningún tipo de respeto a las pertenencias de los demás, no acata ordenes de su autoridad, interrumpe a sus maestros y compañeros, etc. No obstante, cada docente estipula el comportamiento de sus alumnos desde su perspectiva, ciertos maestros atañen el proceder como inquietud natural del estudiante y para otros representa problemas comportamentales e incluso conductuales.

Existen diversos tipos de conducta en los estudiantes. Sin embargo, estas conductas son consideradas cuando muestran excesos y no cuando manifiestan déficits. Por ejemplo Bonilla (2016) manifiesta que los: excesos conductuales (conductas agresivas, violencia, negativismo desafiante e hiperactividad) y déficits conductuales: (aislamiento, cuadros depresivos e introversión).

En cuanto a los excesos y déficits conductuales, la atención se centra en los niños que presentan excesos puesto que llaman más la atención, sin embargo, aunque los déficits llamen la atención, sería importante ser tomados en cuenta por las autoridades.

Es imperativo que existan reglas o normas en toda institución, colectividad, hogar, etc., es decir en cualquier ambiente donde un niño, adolescente, joven o incluso un adulto se desenvuelve. Ya que sin reglas a cumplir no se crea un buen clima y armonía de convivencia social. Por la tanto dentro del aula de clases se debe establecer objetivos comunes de la colectividad, sin embargo, para la consecución de dichos objetivos es muy importante establecer reglas de buen vivir, como lo explica Algara (2016), por ejemplo, los patrones básicos que se deben cumplir son: la puntualidad, el orden, la cordialidad, el respeto, la actitud positiva, la escucha activa, ayuda mutua, etc.

Para facilitar el acatar las reglas escolares, es importante presentar estos reglamentos antes de la matrícula escolar, por lo tanto, los usuarios son conscientes que existen reglas a cumplir y conocen cuales son los lineamientos a seguir.

Es totalmente normal que un estudiante demuestre su comportamiento dentro y fuera del aula de clases, no obstante, ciertos estudiantes cambian sus comportamientos de acuerdo al contexto 
donde se encuentran, y es aquí donde el maestro debe intervenir puesto que juega un papel importante, es imperativo prestarles mayor atención para evitar "malos" entes sociales futuros. Se puede poner de ejemplo, Bonilla (2016) señala algunas técnicas que pueden ser utilizadas para la detección y el mejoramiento del comportamiento estudiantil: conservar el orden en el aula y en tiempos libres intra-jornada, respetar los turnos y las formaciones, hacer grupos cuando el docente lo disponga, poner la basura en su lugar, etc.

Existen muchas actividades que deben considerarse para aplicar a los estudiantes que presentan comportamientos erróneos. El diagnóstico llega a ser perceptible subjetivamente, sin embargo, se debe realizar un trabajo en conjunto con un profesional especializado a diagnósticos comportamentales en estudiantes, y si es necesario remitirlo a profesionales para que se trabaje en los comportamientos psicopedagógicos.

\section{Metodología}

El estudio se desarrollo con un enfoque cualitativo, describiendo que se "Utiliza la recolección y análisis de los datos para afinar las preguntas de investigación o revelar nuevas interrogantes en el proceso de interpretación" (Hernández-Sampieri, Fernández y Baptista, 2014, p.7). Comprendiendo, que el fin de la investigación es obtener datos cualitativos que aporte a conocer las respuestas a las interrogantes del estudio.

Se realizó investigación de campo, para el proceso de recolección de datos la técnica más apropiada es la recolección de datos en campo, se realiza en el lugar donde se planteó la investigación, se realizó la investigación en la modalidad abierta del centro de idiomas de la Universidad Técnica de Ambato.

Además, se empleó un tipo de investigación conocido como bibliográfico documental, en otras palabras, la información se obtiene de, artículos científicos, libros académicos, publicaciones, entre otros., información obtenida con fundamento específico que permitieron determinar conceptos, enfoques, teorías y criterios de sus autores, colaborando con la estructura de este documento.

La presente investigación cuenta con un alcance descriptivo en el que se puntualiza la situación, hechos o procesos con el objetivo de especificar las propiedades o características del grupo investigado. (Rivadeneira, 2015).

En lo expuesto por Hernández-Sampieri, Fernández y Baptista (2014) no se aplicó una muestra probabilística, debido a que los elementos de estudio no se encuentran en dependencia de la probabilidad, por ello, la muestra fueron los estudiantes del idioma ingles del nivel B1+ de la Universidad Técnica de Ambato de la modalidad abierta

\section{Instrumentos}

Se utilizó la Escala de Estilos de Socialización Parental "ESPA29", escala desarrollada y establecida por Musitu y García en el año 2001, la escala puede ser aplicada de forma individual o colectiva en dependencia a la necesidad del estudio, el instrumento está constituido con 29

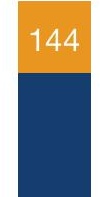


ítems correspondientes a la dimensión de aceptación/implicación y coerción/imposición. La escala está comprendida por 16 situaciones positivas y 13 situaciones negativas valorando a la madre y al padre, las opciones de respuesta van de 1 a 4, con interpretación de Nunca (1), Algunas veces (2), muchas veces (3), y siempre (4); la aplicación de esta escala determinará el estilo de sociabilización parental entre autorizativo, autoritario, indulgente o negligente (Torres, 2016) cross-cutting and correlation type, in order to determine if there is association between the varying styles of parental socialization and social skills in a sample consisting by 177 students of both genders from 12 to 18 years old in a Particular educational institution in Metropolitan Lima. Parental socialization styles questionnaire was used in adolescents (ESPA 29.

La confiabilidad de la escala a través del Alfa de Cronbach con un valor de 0.968 validando el instrumento mediante un análisis factorial exploratorio y confirmatorio.

"TOCA-R", Teacher Observation of Classroom Adaptation instrumento establecido por Werthamer Larsson, Kellam, y Wheeler en el año de 1991, tiene la finalidad de detallar el comportamiento de los estudiantes en el aula, de igual forma, la funcionalidad en el cumplimiento de tareas asignadas. Involucra la ejecución de una entrevista estructurada con el docente. Los docentes clasifican la adaptación del alumno en una escala de Likert entre 1 y 6 , donde 1 es casi nunca y 6 es casi siempre. La mayoría de las interrogantes son de conductas negativas, no obstante, también se cuenta con items de comportamiento positivo. El instrumento cuenta con 43 preguntas.

\section{Resultados}

A continuación, se describe los datos socio demográficos del estudio, se evaluó a 60 estudiantes de la modalidad abierta del idioma inglés, en donde, el $67 \%$ son mujeres y el $33 \%$ son hombres. Las edades correspondientes a cada género y las frecuencias se describen en la siguiente tabla, ver tabla $\mathrm{N}^{\circ} 1$.

Tabla 1. Datos socio demográficos

\begin{tabular}{llllll}
\hline Genero & $\begin{array}{l}\text { Edad } \\
\text { Mujeres }\end{array}$ & $\begin{array}{l}\text { Frecuencia } \\
\text { Mujeres }\end{array}$ & Genero & $\begin{array}{l}\text { Edad } \\
\text { hombres }\end{array}$ & $\begin{array}{l}\text { Frecuencia } \\
\text { hombres }\end{array}$ \\
\hline \multirow{6}{*}{ Femenino } & 13 años & 6 & & 13 años & 3 \\
& 14 años & 10 & & 14 años & 3 \\
& 15 años & 3 & Masculino & 15 años & 2 \\
& 16 años & 10 & & 16 años & 6 \\
& 17 años & 7 & 17 años & 4 \\
& 18 años & 4 & 18 años & 2 \\
\hline
\end{tabular}

Fuente: Datos socio demográficos Autoría propia

En la figura 1 y 2 se detalla de forma gráfica los resultados de los estilos de socialización parental identificados tanto del padre como de la madre. 


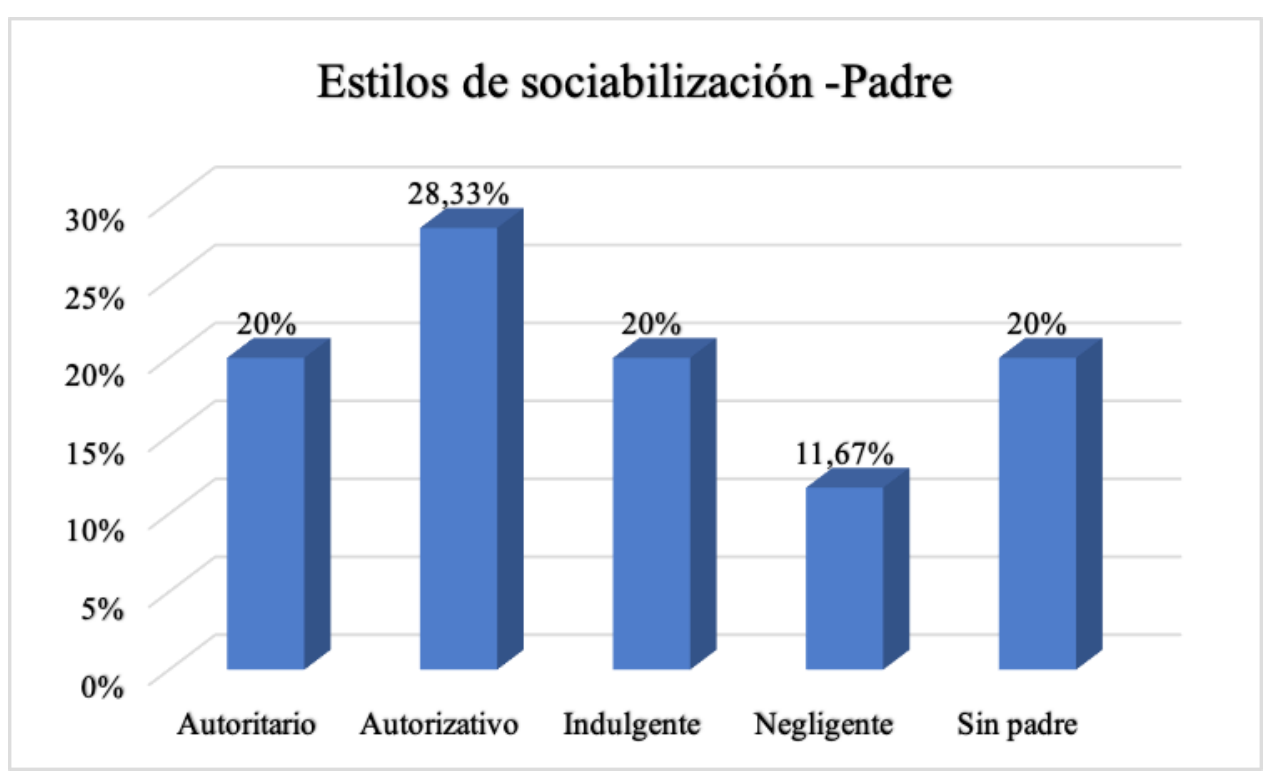

Figura 1. Resultados de los estilos de socialización parental Autoría propia

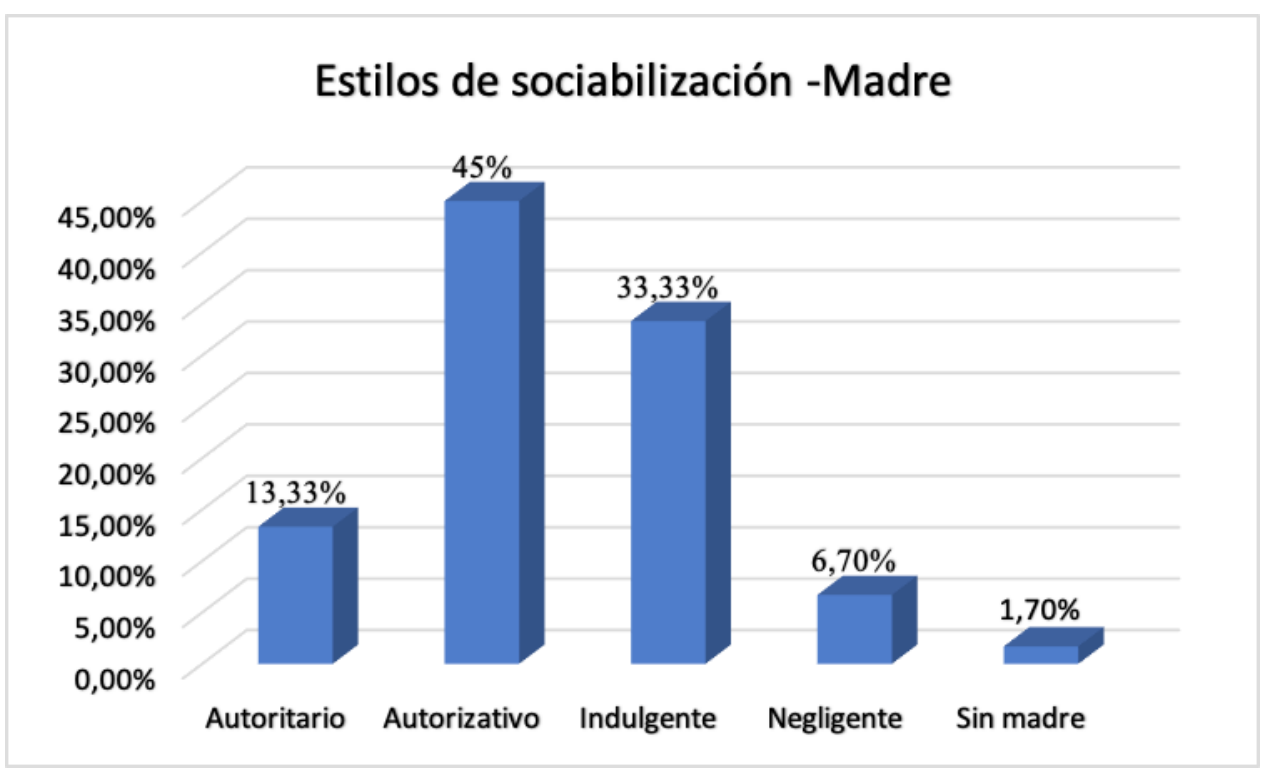

Figura 2. Resultados de los estilos de socialización parental Autoría propia

De los 60 estudiantes evaluados se evidencia en los estilos de la madre predomina el autorizativo (45\%), del mismo modo, existe una predominancia del $28 \%$ del estilo autorizativo, el estilo se caracteriza por el diálogo que existe entre los hijos y sus padres, considerando a la comunicación como un recurso para llegar acuerdos, expresar y escuchar opiniones; existe una similitud de una baja presencia del estilo negligente en la madre (7\%), se considera también, que en el padre existe una presencia baja pero considerable debido a que es superior al $20 \%$, estimando que existe un 
compromiso hacia el control de los hijos, sin establecer límites o normas ni.

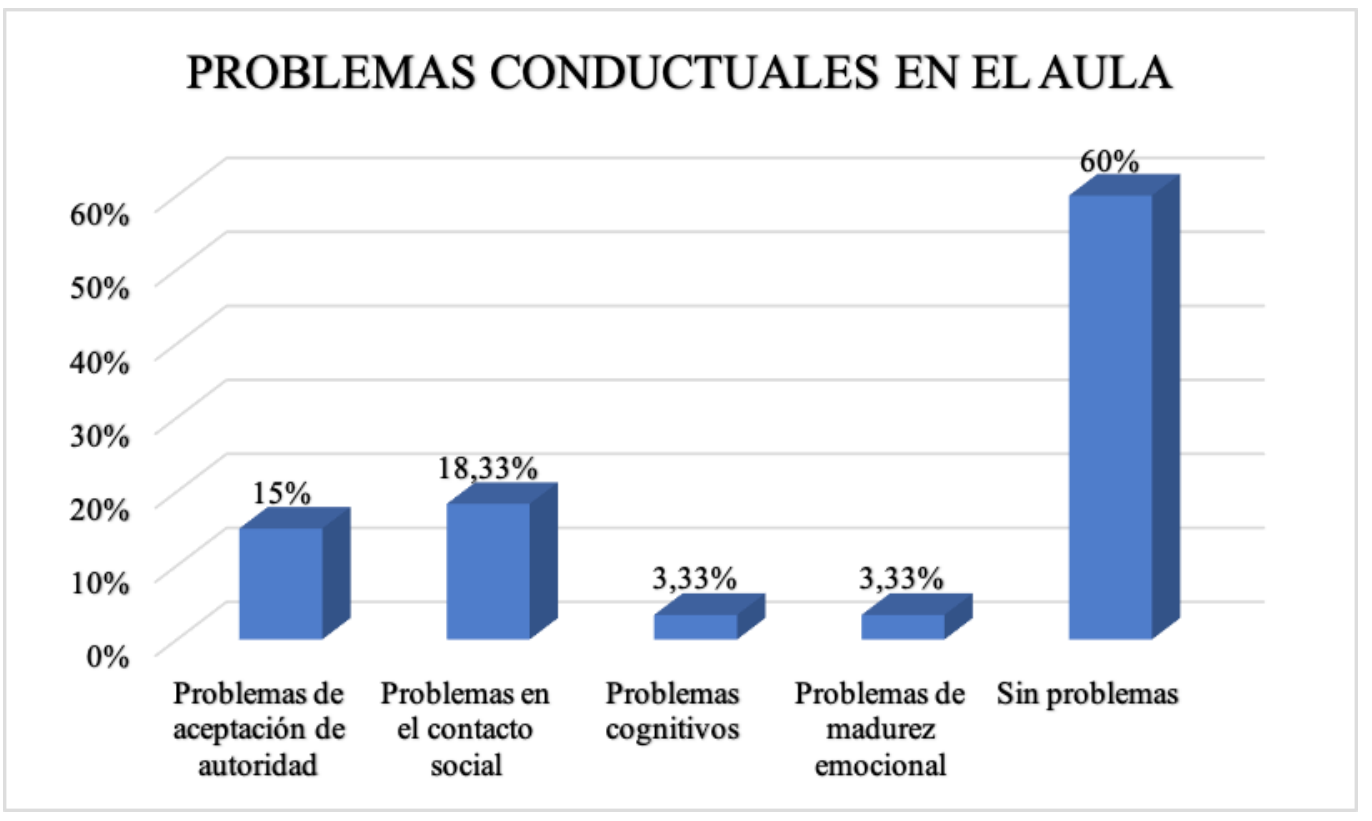

Figura 3. Resultados de los estilos de socialización parental

Autoría propia

De los estudiantes evaluados se evidencia que el $60 \%$ no presenta ningún problema conductual, por otra parte, el $18.33 \%$ a presentado problemas en el contacto social, continuando con la presencia de problemas de aceptación de autoridad se encuentra el 15\%, para finalizar, se encuentran los problemas cognitivos y de madurez emocional con el $3.33 \%$.

En función a los resultados se entiende que no existe presencia predominante de conductas negativas, a pesar de ello, existe la presencia de problemas en el contacto social, entendiendo que la persona es poco amistosa, es importante, mencionar que existe problemas de aceptación de autoridad, expresándose a través de acciones como desobediencia y en varios casos la agresión

\section{Conclusiones}

Al pretender conocer los estilos de socialización parental en los estudiantes del idioma ingles se concluye que existe los cuatro estilos con diferenciación en los niveles de presencia tanto en la madre como en el padre, no obstante, existe una diferencia en la presencia del estilo autorizativo con un nivel mayor de presencia, donde se interpreta que existe una relación entre padres y estudiantes basado en el dialogo y una buena comunicación

Por otra parte, se buscó identificar la presencia de problemas conductuales en la que una parte considerable de los estudiantes no tiene ningún problema de conducta, sin embargo, de forma negativa existe la presencia de problemas de contacto social y de aceptación de autoridad en mayor nivel obteniendo así factores de riego como agresión, desobediencia y conducta tímida en 
el aula de clases.

Los resultados se encuentran fundamentados por instrumentos de evaluación direccionados a las variables de estudio, se estima que tanto los problemas conductuales como el estilo parental influye en el proceso de aprendizaje del idioma ingles debido a que los factores de constante presencia en los estudiantes. 


\section{Referencias Bibliográficas}

Adame, D., García, N., \& Gómez, N. (2017). Ambiente escolar; convivencia y paz. Universidad Pontificia Bolivariana de Medellín.

Aguinaga, S., Rimari, M., \& Valázquez, M. (2018). Modelo contextualizado de inclusión educativa. Revista Educación, 42(2), 1-35. https://doi.org/https://doi.org/10.15517/revedu.v42i2.23885

Algara, A. (2016). Los acuerdos del aula una estrategia de convivencia para fortalecer la democracia en la escuela primaria. Ra Ximhai, 12(3), 207-214. https://doi.org/10.35197/rx.12.02.2016.13.aa

Bonilla, J. (2016). El rol del docente y su influencia en el comportamiento de los estudiantes del quinto grado de la escuela particular N 12 "Sergio Núñez Santamaría", Parroquia Posorja, cantón Guayaquil, período lectivo 2015-2016. Universidad Estatal Península de Santa Elena.

Brea, L. (2014). Factores determinantes del sentido de pertenencia de los estudiantes de Arquitectura de la Pontifica Universidad Católica Madre y Maestra, Campus Santo Tomás de Aquino. Universidad de Murcia.

Capano, A., González, M. del L., \& Massonnier, N. (2016). Estilos relacionales parentales: estudio con adolescentes y sus padres. Revista de Psicología, 34(2), 413-444. https://doi.org/10.18800/psico.201602.008

Capano, Á., \& Ubach, A. (2013). Estilos parentales, parentalidad positiva y formación de padres. Ciencias Psicológicas, 7(1), 83-95.

Carvajal, M. F. (2015). El rol parental y su incidencia en el comportamiento socio-afectivo de los estudiantes de educación general básica superior de la Unidad Educativa Particular "Bautista" de la ciudad de Ambato, Provincia de Tungurahua. Universidad Técnica de Ambato.

Chávez, M. (2013). La familia, las relaciones afectivas y la identidad étnica entre indígenas migrantes urbanos en San Luis Potosí. Relaciones: Estudios de Historia y Sociedad, 34(134), 131-155.

Comino, M., \& Raya, A. (2014). Estilos educativos parentales y su relación con la socialización en adolescentes. Apuntes de Psicología, 32(3), 271-280.

Córdoba, J. (2014). Estilo de criaza vinculados a comportamientos problematicos de niñas, niños y adolescentes. Universidad Nacional de Córdoba. Retrieved from http://lildbi.fcm.unc.edu.ar/lildbi/tesis/cordoba_julia. pdf

Erazo, O. (2016). La adaptabilidad en el aula: Una reflexión desde los trastornos de integración sensorial, atención y conducta. Tesis Psicológica, 11(2), 36-52.

Espinoza, C., \& Panta, C. (2014). Estilos de socialización parental y asertividad en las estudiantes de acuerto 
año de secundaria de una institución educativa. Revista USS, 5(1), 11. Retrieved from http://revistas.uss. edu.pe/index.php/PAIAN/article/download/226/239/

Espinoza, Y. (2015). Estudios dobre la disfuncionalidad familiar y su incidencia en el aprendizaje de los niños y niñas de primero y segundo año de educación primaria de la escuela Sagrado Corazón de Jesús de Tulcán. Escuela Politécnica Nacional. Retrieved from https://bibdigital.epn.edu.ec/bitstream/15000/10577/1/ CD-6255.pdf

Fuentes, M., García, F., Gracia, E., \& Alarcón, A. (2015). Los estilos parentales de socialización y el ajuste psicológico. Un estudio con adolescentes españoles. Revista de Psicodidactica, 20(1), 117-138. https://doi. org/10.1387/RevPsicodidact.10876

George, M., Squicciarini, A., \& Zapata, R. (2004). Detección Precoz de Factores de Riesgo de Salud Mental en Escolares. Revista de Psicología Dela Universidad de Chile, 13(2), 9-20.

Gotzens, C., Badía, M. M., Castelló, A., \& Genovar, C. (2007). La gravedad de los problemas de comportamiento en el aula vista por los profesores. Revista Portuguesa de Pedagogia, 41(1), 103-120. Retrieved from http://dspace.ucuenca.edu.ec/bitstream/123456789/27326/1/Trabajo de Titulación.pdf

Guevara, J. (2013). Ecología humana y acción pro-ambiental: Alteridades recíprocas aula-escuelacomunidad para el manejo sustentable de residuos. Revista Latinoamericana de Psicologia, 45(3), 449-459. https:// doi.org/10.14349/rlp.v45i3.1486

Hernández-Sampieri, R., Fernández, C., \& Baptista, M. (2014). Metodología de la Investigación (6ta ed.). México, D.F.: McGraw-Hill.

Huerta, A. (2018). El sentido de pertenencia y la identidad como determinante de la conducta, una perspectiva desde el pensamiento complejo. IE Revista de Investigación Educativa de La REDIECH, 9(16), 83-97.

López, K. (2014). Estilos de socialización parental en adolescentes indígenas Kaqchikeles. Implementation Science. Universidad Rafael Landívar. https://doi.org/10.4324/9781315853178

Marmo, J. (2014). Estilos parentales y factores de riesgo asociados a la patología alimentaria. Unife.Edu.Pe, 22(2), 165-178.

Oliva, E., \& Villa, V. (2014). Hacia un concepto interdisciplinario de la familia en la globalización. Justicia Juris, 10(1), 11. https://doi.org/10.15665/rj.v10i1.295

Ortega, P., Plancarte, P., Garrido, A., Reyes, A., \& Torres, L. (2018). Una visión de alumnos universitarios sobre la relación de pareja de sus padres. Acta Colombiana de Psicología, 21(2), 88-109. https://doi.org/10.14718/ acp.2018.21.2.5 
Ortiz, M., \& Moreno, O. (2016). Los estilos parentales: implicaciones sobre el rendimiento escolar en alumnos de educación media. Revista Digital Internacional de Psicología y Ciencia Social, 2(1), 76-94.

Páramo, D. (2017). Cultura y comportamiento humano. Revista Científica Pensamiento y Gestión, (42), 7-11. https://doi.org/10.14482/pege.42.10450

Pérez, O. (2013). Socialización parental en la adolescencia. Universidad Rafael Landívar. Retrieved from http:// biblio3.url.edu.gt/Tesario/2013/05/24/Perez-Orlando.pdf

Rivadeneira, E. (2015). Comprensión teórica y proceso metodológico de la investigación cualitativa. In Crescendo, 6(2), 169-183. https://doi.org/10.21895/incres.2015.v6n2.16

Suárez, P., \& Vélez, M. (2018). El papel de la familia en el desarrollo social del niño : una mirada desde la afectividad, la comunicación. Psicoespacios, 12(20), 173-197.

Torres, V. (2016). Estilos de socialización parental y habilidades sociales en estudiantes del nivel secundario de una institución educativa particular de Lima metropolitana, 2015. Universidad Peruana Union. 
DIEGO BONILLA · DANNY HALLO · GIOMARA QUIZHPE · CARLOS TACO

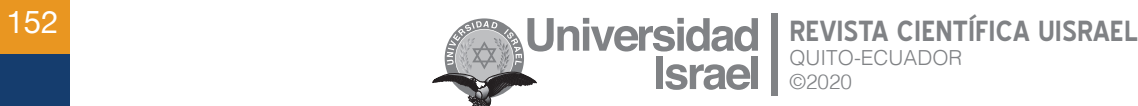

